\title{
Aperiodic Model Predictive Control via Perturbation Analysis
}

\author{
Alina Eqtami, Dimos V. Dimarogonas and Kostas J. Kyriakopoulos
}

\begin{abstract}
In this paper, an enhanced event-based scheme for model predictive control (MPC) of constrained discretetime systems with additive disturbances is investigated. The re/calculation of the MPC control law is triggered whenever an event depending on the error of the measured state with respect to the nominal state of the system occurs. Between the controller updates, the last computed control trajectory is applied to the system, in conjunction with a correction term. This term consists of a perturbation solution of the nominal system which itself depends on the aforementioned error. The overall framework yields less conservative results with respect to our previous work. The results are illustrated through a simulated example.
\end{abstract}

\section{INTRODUCTION}

The formulation of event-based control schemes is a flourishing field in the recent years. The key attribute of these approaches is that the decision for the execution of the control task is not made ad-hoc, but it is based on a certain condition of the state of the system. This results to a more flexible aperiodic sampling, while preserving necessary properties of the system such as stability and convergence. The event-based methodology may lead to an overall reduction on the number of the control updates which might be desirable when the system has limited resources. Indicatively, related works on event-based control can be found in [1], [4], [7], [11], [15], [18], [20].

The NMPC strategy is a widely used control strategy for constrained systems. Even though formulating a control problem under NMPC is intuitively attractive, the computation of the control law is considerably demanding. Motivated by this fact, an event-based framework for this kind of controllers has been investigated, in order to reduce the number of times the control input should be computed. This event-based approach exploits the fact that predictive controllers provide a control sequence for a prediction horizon. The main idea is that the control sequence provided by the controller is applied to the system in an open-loop fashion between actuator updates. Sufficient conditions for triggering the predictive control law of an uncertain discretetime nonlinear system has already been presented in our previous work, [5].

The problem addressed here is the event-driven control of a general nonlinear discrete-time system with additive

Alina Eqtami and Kostas J. Kyriakopoulos are with the Control Systems Lab, Department of Mechanical Engineering, National Technical University of Athens, 9 Heroon Polytechniou Street, Zografou 15780, Greece $\{a l i n a, k k y r i a @ m a i l . n t u a . g r\}$. Dimos V. Dimarogonas is with the ACCESS Linnaeus Centre, School of Electrical Engineering, KTH Royal Institute of Technology, Stockholm, Sweden. He is also affiliated with the KTH Centre for Autonomous Systems and is supported by the Swedish Research Council (VR) through contract 20093948. \{dimosdee.kth.se\}. disturbances, under an NMPC framework. Since the system in consideration is uncertain, in order to prove stability, a similar procedure as in the case of Input-to-State (ISS) stability analysis for MPC, is going to be used. Some relevant citations of ISS MPC schemes are [13], [14], [16]. As in [5], the error between the real state of the system and the predicted state given by the nominal model, is monitored. However, the control law that is applied to the plant, during the inter-event times, is the previously computed control law in conjunction with a correction term. This term utilizes a perturbation solution of the optimal trajectory and is explicitly dependent on the aforementioned error. With this approach the controller has some kind of additional "intelligence" and reacts to the measured error. Thus, the contribution of this paper relies in finding sufficient conditions for triggering in the case of uncertain discrete-time systems under an NMPC control law with a correction term. Notice, that perturbation analysis of predictive controllers has been presented in [8], [9] and [21].

The field of event-based MPC is quite new, however, some relevant works have already been presented. Some relevant papers are [2], [10], [12], [17], [19].

The remainder of the paper is organized as follows. In Section II, the system under consideration is presented. Subsequently, the problem formulation for the event-based control of uncertain nonlinear systems under NMPC control law along with a correction term is given. A brief analysis on the neighboring extremals is conducted first and then the convergence and stability proofs of the overall scheme are provided in Section III. In Section IV, sufficient conditions for triggering are designed, while in Section V some simulation results are presented. Finally, Section VI summarizes the results of this paper and indicates future research goals.

\section{PRoblem Formulation}

Consider the nonlinear discrete-time dynamic system

$$
x_{k+1}=f\left(x_{k}, u_{k}\right)
$$

where $x_{k} \in \mathbb{R}^{n}$ denotes the system's state and $u_{k} \in \mathbb{R}^{m}$ is the control vector. The state and control variables are subject to the following constraints

$$
x_{k} \in X, \quad u_{k} \in U, \quad k \in \mathbb{Z}_{\geq 0}
$$

where $X$ is a closed subset of $\mathbb{R}^{n}$ and $U$ is a compact subset of $\mathbb{R}^{m}$, both of them containing the origin as an interior point. Assume that $f(0,0)=0$ and that $f(x, u)$ is locally Lipschitz with respect to $x$ and $u$ in the domain $X \times U$, with Lipschitz constants $L_{f_{x}}$ and $L_{f_{u}}$, respectively. The predicted state of the system at a time step $k+j+1$ with $j \in \mathbb{Z}_{\geq 0}$ can be found by 
the nominal model of the system i.e. (1), and is denoted as $\hat{x}(k+j+1 \mid k)=f\left(\hat{x}(k+j \mid k), u_{k+j}\right)$, where $u_{k+j}$ is a control sequence for time $[k, k+j]$ and $x_{k}=\hat{x}(k \mid k)$ is the measured state of the system at time step $k$.

In a realistic formulation though, modeling errors, uncertainties and disturbances may exist. Thus, a perturbed version of (1) is going to be considered as well. The perturbed system is described as

$$
x_{k+1}=f\left(x_{k}, u_{k}\right)+w_{k}
$$

with $w_{k} \in W \subseteq \mathbb{R}^{n}$ to be the additive disturbance and $W$ to be a compact set containing the origin. The admissible set of uncertainties are bounded, thus

$$
w_{k} \in W, \quad\left\|w_{k}\right\| \leq \gamma
$$

It is apparent that the uncertainty of system (3) can cause discrepancies between the predicted state sequence given from (1) and the actual state sequence of the system. This divergence can be quantified in terms of an error. Therefor, the error $e(k+j \mid k)$ is introduced in the analysis and is denoted as

$$
e(k+j \mid k)=\left\|x_{k+j}-\hat{x}(k+j \mid k)\right\|
$$

\section{EVENT-BASED NMPC VIA PERTURBATION ANALYSIS}

In the classic NMPC strategy, the control law is updated at each time-step $k$. The control input that is applied to the system is the first term of the optimal control sequence provided by the NMPC. However, in the event-triggered setup the rest of the optimal sequence might be used as well, provided that the real evolution of the system stays close to the predicted by means of the nominal model. In our previous work [5], the last computed control sequence was applied to the system in an open-loop fashion, during the inter-event times. The error between the real state sequence and the predicted sequence was monitored in order to trigger an event. On the other hand, in this approach, the last computed control law along with a correction term is applied to the system during the triggering events. The correction term can be found as the approximation solution of the MPC, it is easily computable and corrects the nominal solution. Hence, this term is applied in order to account for the error on-line.

In the following, a perturbation analysis is conducted in order to reach to the the analytic expression of the correction term. Moreover, the convergence and stability properties of the overall scheme of the NMPC with the neighboring extremals approach are proven.

\section{A. Neighboring Extremals}

The solution of an optimal control problem, when perturbations in the initial state are present, can be approximated using the optimal perturbation analysis approach. Namely, if there is a perturbation $d x(k)$ in the initial condition, the resulting optimal solution can be approximated by $\hat{x}(k)+$ $d x(k)$ and $u^{*}(k)+d u(k)$. The real state of the system $x_{k}$ at time step $k$, can be found by

$$
x_{k}=\hat{x}(k \mid k-1)+d x(k) \Rightarrow d x(k)=x_{k}-\hat{x}(k \mid k-1)
$$

and for time step $k+j$ we have respectively $d x(k+j)=$ $x_{k+j}-\hat{x}(k+j \mid k-1)$.

The neighboring extremal path method, developed in [3], is adopted. It holds that

$$
d u(k+j)=K^{*}(k+j) d x(k+j)
$$

In order to find $K^{*}(k+j)$, all quantities are evaluated at the nominal optimal condition, namely, $\hat{x}(k+j \mid k-1), u^{*}(k-1)$. The analytic expression of $K^{*}(k+j)$ is derived in the Appendix and in particular in (29). From (7) we have

$$
\begin{aligned}
& \|d u(k+j)\| \leq\left\|K^{*}(k+j)\right\| \cdot\|d x(k+j)\| \\
& \leq\left\|K^{*}(k+j)\right\| e(k+j \mid k-1)
\end{aligned}
$$

Suppose an upper bound on $\|d u(\cdot)\|$. This is a because the system (1) is constrained in the inputs, so we must ensure that while using the neighbors extremals, the input constraints will be fulfilled. So,

$$
\|d u(k+j)\| \leq \gamma^{u}
$$

\section{B. NMPC Strategy}

The general form of NMPC consists in solving on-line a finite-horizon, open-loop optimal control problem (abbr. OCP), based on the current state measurement. A cost function $J_{N}$ is minimized with respect to a control sequence $u_{F}(k) \triangleq[u(k \mid k), u(k+1 \mid k), \ldots, u(k+N-1 \mid k)]$, thus, the OCP for the nominal system (1), can be formulated as follows

$$
\begin{aligned}
& \min _{u_{F}(k)} J_{N}\left(x_{k}, u_{F}(k)\right)= \\
& \min _{u_{F}(k)} \sum_{i=0}^{i=N-1} F(\hat{x}(k+i \mid k), u(k+i \mid k))+V(\hat{x}(k+N \mid k))
\end{aligned}
$$

s.t.

$$
\hat{x}(k+j \mid k) \in X_{j} \quad u(k+j \mid k) \in U_{j} \quad \hat{x}(k+N \mid k) \in X_{f}
$$

for all $j=0, \ldots, N-1$. The positive integer $N \in \mathbb{Z}_{\geq 0}$ denotes the prediction horizon and $X_{f}$ denotes the terminal constraint set.

The constraints on the state from (2) are being replaced by a restricted constraint set $X_{j}$ while solving the OCP. It holds that $X_{j}=X \sim \mathscr{B}_{j}^{x}$ where $\mathscr{B}_{j}^{x}=\left\{x \in \mathbb{R}^{n}:\|x\| \leq\right.$ $\left.L_{f_{x}}^{j} \gamma+L_{m}(j) L_{f_{u}} \gamma^{u}\right\}$. This state constraints' tightening for the nominal system with additive disturbances, while utilizing the correction term from the perturbation analysis, guarantees that the evolution of the real system will be admissible for all time. This is proven in Lemma 4 of the Appendix. Furthermore, the constrained set $U_{j}$ is a restricted set in the same sense as in the state constraint tightening case. There is $U_{j}=U \sim \mathscr{B}^{u}$ where $\mathscr{B}^{u}=\left\{u \in \mathbb{R}^{m}:\|u\| \leq \gamma^{u}\right\}$ which guarantees the fulfillment of all input constraints. Notice that, the set operator " $\sim$ " denotes the Pontryagin difference and that we denote

$$
L_{m}(j)=\sum_{i=0}^{j-1}\left\{L_{f_{x}}^{i}\right\}
$$

Similarly to [14], the following assumptions for the stage cost $F(\cdot)$ and the terminal cost $V(\cdot)$ are stated: 
Assumption 1: i) The stage cost $F(x, u)$ is Lipschitz continuous with respect to $x$ and $u$ in $X \times U$, with Lipschitz constants denoted by $L_{F x}$ and $L_{F u}$, respectively. Assume that $F(0,0)=0$ and that there are positive constants $\alpha>0$ and $\omega \geq 1$, such that $L(x, u) \geq \alpha\|(x, u)\|^{\omega}$.

ii) Let the terminal region $X_{f}$ from (10b) be a subset of an admissible positively invariant set $\Phi$ of the nominal system. Assume that there is a local stabilizing controller $h\left(x_{k}\right)$ for the terminal state $X_{f}$. The associated Lyapunov function $V(\cdot)$ has the following properties $V\left(f\left(x_{k}, h\left(x_{k}\right)\right)-V\left(x_{k}\right) \leq\right.$ $-F\left(x_{k}, h\left(x_{k}\right)\right), \forall x_{k} \in \Phi$, and is Lipschitz in $\Phi$, with Lipschitz constant $L_{V}$. The set $\Phi$ is given by $\Phi=\left\{x_{k} \in \mathbb{R}^{n}: V\left(x_{k}\right) \leq\right.$ $\left.\alpha_{\Phi}\right\}$ such that $\Phi \subseteq X^{h}=\left\{x_{k} \in X_{N-1}: h\left(x_{k}\right) \in U\right\}$. The set $X_{f}=\left\{x_{k} \in \mathbb{R}^{n}: V\left(x_{k}\right) \leq \alpha_{v}\right\}$ is such that for all $x_{k} \in \Phi$, $f\left(x_{k}, h\left(x_{k}\right)\right) \in X_{f}$.

Definition 1: In the following, $X^{M P C}$ will denote the set containing all the state vectors for which a feasible control sequence exists, i.e. a control sequence $u$ that satisfies all the constraints of the MPC (10b).

Consider the control trajectories $u_{F}^{n}(k+m)$, for time steps $m=0, \ldots, N-1$, based on the optimal solution in $k-1$, i.e. $u_{F}^{*}(k-1)$, in conjunction with a correction term from the perturbation solution of the MPC. The "neighboring" control trajectories can be denoted as

$$
\begin{aligned}
& u^{n}(k+j \mid k+m)= \\
& =\left\{\begin{aligned}
u^{*}(k+j \mid k-1)+d u(k+j) & \text { for } j=m, \ldots, N-2 \\
h\left(x^{n}(k+N-1 \mid k+m)\right) & \text { for } j=N-1
\end{aligned}\right.
\end{aligned}
$$

Furthermore, the state of the system when the control law (11) is applied to the system, is given by

$$
x^{n}(k+j+1 \mid k+m)=f\left(x^{n}(k+j \mid k+m), u^{n}(k+j \mid k+m)\right)
$$

Definition 2: A set $X^{M P C} \subseteq X$ is robust positively invariant (RPI) set for system (3), if $x_{k} \in X^{M P C}, \forall x_{k-1} \in X^{M P C}$ and $\forall w_{k} \in W$.

Next, the robust positively invariance of the set $X^{M P C}$ of the closed-loop system will be shown.

Lemma 1: Let the system described by (3) and is subject to (2). Under the Assumption 1, $X^{M P C}$ is RPI for the closedloop system if the uncertainties are bounded by $\gamma \leq\left(\alpha_{\Phi}-\right.$ $\left.\alpha_{v}-L_{V} L_{m}(N-1) L_{f_{u}} \gamma^{u}\right) / L_{V} L_{f_{x}}^{N-1}$.

Proof: For simplicity, we are going to treat the case $m=0$. One can easily verify that $u^{n}(k+j \mid k) \in U_{j}$ for $j \in$ $[0, N-2]$, and $h(x) \in U$, which yields that $u^{n}(\cdot)$ are feasible control trajectories. Also it must be shown that if $\hat{x}(k+N-$ $1 \mid k) \in \Phi$, then $x^{n}(k+N \mid k) \in X_{f}$. By applying Lemma 5 in the Appendix, it holds that

$$
\begin{aligned}
& V\left(x^{n}(k+N \mid k)\right) \leq V(\hat{x}(k+N-1 \mid k-1)) \\
& +L_{V} L_{f_{x}}^{N-1} \gamma+L_{V} L_{m}(N-1) L_{f_{u}} \gamma^{u} \leq \\
& \alpha_{V}+L_{V} L_{f_{x}}^{N-1} \gamma+L_{V} L_{m}(N-1) L_{f_{u}} \gamma^{u} \leq \alpha_{\Phi}
\end{aligned}
$$

Considering that ||$x^{n}(k+j \mid k)-x^{n}(k+j \mid k-1) \| \leq L_{f_{x}}^{j} \gamma+$ $L_{m}(j) L_{f_{u}} \gamma^{u}$, it can be concluded that $x^{n}(k+j \mid k) \in X_{j}$, and the proof is completed.

The next step is to prove convergence of the proposed scheme. In order to do so, an intermediate result is going to be stated first. The optimal cost at time step $k-1$ is $J_{N}^{*}(k-1)$ and the cost of the "neighboring" feasible sequence at a time step $j \in[0, N-1]$ is indicated by $J_{N}^{n}(k+j)$. Then the difference of these costs is

$$
\Delta J_{j}=J_{N}^{n}(k+j)-J_{N}^{*}(k-1)
$$

The next theorem can now be stated:

Theorem 2: Consider the system (3) subject to (2) and assume that the previously presented Assumption 1 holds. Then, using the control law from (11), the difference between the cost of a feasible sequence at time step $k+j$ and the optimal cost of at time step $k-1$ is bounded by

$$
\Delta J_{j} \leq C_{1}^{j} e(k+j \mid k-1)-\alpha \sum_{i=0}^{j}\left\{|| x_{k-i+j} \|^{\omega}\right\}+C_{2}^{j}
$$

where $C_{1}^{j}$ is given by

$$
C_{1}^{j} \triangleq L_{F_{x}} L_{m}(N-1-j)+L_{V} L_{f_{x}}^{N-1-j}
$$

and $C_{2}^{j}$ is given by

$$
C_{2}^{j} \triangleq\left(L_{V} L_{m}(N-1-j)+L_{F_{x}} \sum_{i=0}^{N-2-j}\left\{L_{m}(i)\right\}+1\right) L_{f_{u}} \gamma^{u}
$$

Proof: First, the difference (12) is calculated for $j=0$. Then the calculation will be repeated for $j=1$, and finally the general rule for random $j$ will be stated.

For $j=0$ the difference (12) is

$$
\begin{aligned}
& \Delta J_{0}=J_{N}^{n}(k)-J_{N}^{*}(k-1)=\sum_{i=0}^{N-1}\left\{F\left(x^{n}(k+i \mid k), u^{n}(k+i \mid k)\right)\right. \\
& \left.-F\left(\hat{x}(k+i-1 \mid k-1), u^{*}(k+i-1 \mid k-1)\right)\right\}+V\left(x^{n}(k+N \mid k)\right) \\
& -V(\hat{x}(k+N-1 \mid k-1))=\sum_{i=0}^{N-2}\left\{F\left(x^{n}(k+i \mid k), u^{n}(k+i \mid k)\right)\right. \\
& \left.-F\left(\hat{x}(k+i \mid k-1), u^{*}(k+i \mid k-1)\right)\right\} \\
& +F\left(x^{n}(k+N-1 \mid k), h\left(x^{n}(k+N-1 \mid k)\right)-F\left(x_{k-1}, u_{k-1}\right)\right. \\
& +V\left(x^{n}(k+N \mid k)\right)-V(\hat{x}(k+N-1 \mid k-1)) \\
& +V\left(x^{n}(k+N-1 \mid k)\right)-V\left(x^{n}(k+N-1 \mid k)\right)
\end{aligned}
$$

Recall from Assumption 1, that the stage cost is Lipschitz continuous in $X \times U$, so

$$
\begin{aligned}
& F\left(x^{n}(k+i \mid k), u^{n}(k+i \mid k)\right)-F\left(\hat{x}(k+i \mid k-1), u^{*}(k+i \mid k-1)\right) \\
& \leq L_{F x}|| x^{n}(k+i \mid k)-\hat{x}(k+i \mid k-1) \| \\
& +L_{F u}|| u^{n}(k+i \mid k)-u^{*}(k+i \mid k-1) \|
\end{aligned}
$$

From (36) of the Appendix, it can be concluded that

$$
\begin{aligned}
& L_{F_{x}}|| x^{n}(k+i \mid k)-\hat{x}(k+i \mid k-1) \| \leq \\
& L_{F_{x}} L_{f_{x}}^{i} e(k \mid k-1)+L_{F_{x}} L_{m}(i) L_{f_{u}} \gamma^{u}
\end{aligned}
$$

Also, using the control law (11), we have

$$
\begin{aligned}
& L_{F u}|| u^{n}(k+i \mid k)-u^{*}(k+i \mid k-1) \|= \\
& L_{F u}\left\|u^{*}(k+i \mid k-1)+d u(k+i)-u^{*}(k+i \mid k-1)\right\|= \\
& L_{F u}|| d u(k+i) \| \leq L_{F u} \gamma^{u}
\end{aligned}
$$


The following inequality holds by Assumption 1,

$$
\begin{aligned}
& V\left(x^{n}(k+N \mid k)\right)-V\left(x^{n}(k+N-1 \mid k)\right) \\
& +F\left(x^{n}(k+N-1 \mid k), h\left(x^{n}(k+N-1 \mid k)\right)\right) \leq 0
\end{aligned}
$$

Moreover, using (36) it follows that

$$
\begin{aligned}
& V\left(x^{n}(k+N-1 \mid k)\right)-V(\hat{x}(k+N-1 \mid k-1)) \\
& \leq L_{V} L_{f_{x}}^{N-1} e(k \mid k-1)+L_{V} L_{m}(N-1) L_{f_{u}} \gamma^{u}
\end{aligned}
$$

Let the stage cost to be $F(x, u) \geq \alpha\|(x, u)\|^{\omega} \geq \alpha\|x\| \|^{\omega}$. Substituting (18)-(21) to (16), the following is derived

$$
\begin{aligned}
& \Delta J_{0} \leq \sum_{i=0}^{N-2}\left\{L_{F_{x}} L_{f_{x}}^{i} e(k \mid k-1)+L_{F_{x}} L_{m}(i) L_{f_{u}} \gamma^{u}+L_{f_{u}} \gamma^{u}\right\} \\
& +L_{V} L_{f_{x}}^{N-1} e(k \mid k-1)+L_{V} L_{m}(N-1) L_{f_{u}} \gamma^{u} \\
& \leq\left(L_{F_{x}} L_{m}(N-1)+L_{V} L_{f_{x}}^{N-1}\right) e(k \mid k-1) \\
& +\left(L_{V} L_{m}(N-1)+L_{F_{x}} \sum_{i=0}^{N-2}\left\{L_{m}(i)\right\}+1\right) L_{f_{u}} \gamma^{u}-\alpha\left\|x_{k-1}\right\|^{\omega} \\
& \leq C_{1}^{0} e(k \mid k-1)-\alpha\left\|x_{k-1}\right\|^{\omega}+C_{2}^{0}
\end{aligned}
$$

where $C_{1}^{0}, C_{2}^{0}$ are constant terms from (14) and (15) for $j=0$, respectively.

For $j=1$ the difference (12) becomes

$$
\begin{aligned}
& \Delta J_{1}=J_{N}^{n}(k+1)-J_{N}^{*}(k-1) \\
& =\sum_{i=0}^{N-1}\left\{F\left(x^{n}(k+i+1 \mid k+1), u^{n}(k+i+1 \mid k+1)\right)\right. \\
& \left.-F\left(\hat{x}(k+i-1 \mid k-1), u^{*}(k+i-1 \mid k-1)\right)\right\} \\
& +V\left(x^{n}(k+N+1 \mid k+1)\right)-V(\hat{x}(k+N-1 \mid k-1)) \\
& \leq\left(L_{F_{x}} L_{m}(N-2)+L_{V} L_{f_{x}}^{N-2}\right) e(k+1 \mid k-1) \\
& +\left(L_{V} L_{m}(N-2)+L_{F_{x}} \sum_{i=0}^{N-3}\left\{L_{m}(i)\right\}+1\right) L_{f_{u}} \gamma^{u} \\
& -\alpha\left\|x_{k-1}\right\|^{\omega}-\alpha\left\|x_{k}\right\|^{\omega} \\
& \leq C_{1}^{1} e(k+1 \mid k-1)+C_{2}^{1}-\alpha\left\|x_{k-1}\right\|^{\omega}-\alpha\left\|x_{k}\right\|^{\omega}
\end{aligned}
$$

From the above it can be concluded using the same calculation, that for random $j \in[0, N-1]$ the difference $\Delta J_{j}=J_{N}^{n}(k+j)-J_{N}^{*}(k-1)$, is given from (13), and hence the proof is completed.

\section{TRiggering CONDition}

The proposed scheme must be convergent to a compact set where the system is ultimately bounded. In the following, a triggering condition for the OCP of the MPC that guarantees that the associate Lyapunov function is decaying at every time step and that all the constraints are fulfilled, is given.

Since $J_{N}^{*}(k)$ is the optimal cost at time step $k$, we have $J_{N}^{*}(k)-J_{N}^{*}(k-1) \leq \Delta J_{0}$, hence

$$
J_{N}^{*}(k)-J_{N}^{*}(k-1) \leq C_{1}^{0} e(k \mid k-1)-\alpha\left\|x_{k-1}\right\|^{\omega}+C_{2}^{0}
$$

The triggering condition is written in this case as

$$
C_{1}^{0} e(k \mid k-1) \leq \sigma\left(\alpha|| x_{k-1} \|^{\omega}-C_{2}^{0}\right)
$$

Invoking this rule into (24), with $0<\sigma<1$, it can be concluded that $J_{N}^{*}(\cdot)$ is strictly decreasing. The triggering rule (25) is valid, though, only in the first step. In order to maintain stability we must ensure that $\Delta J_{j}$ is strictly decreasing for all $j \in[0, N-1]$. The control law (11), is applied to the system as long as

$$
\Delta J_{j+1} \leq \Delta J_{j}
$$

In this case the convergence of the closed-loop system is guaranteed.

As we have already discussed, correcting the optimal control law with an approximation solution given by the neighboring extremals approach, may lead to control inputs that violate the constraints. To account for that, an upper bound on the norm of the correction term was assumed in (9). In order to assert that this is the case, we will impose an event-based condition that states that the control update will be triggered whenever the tracking error will exceed a specific limit. Using (7) and (9) the following condition can be derived

$$
\left\|K^{*}(k+j)\right\| e(k+j) \leq \gamma^{u}
$$

In a practical sense, this states that whenever the error is small enough we do not trigger a new MPC law, otherwise, we measure the state of the system and compute an appropriate control law.

Consequently, the triggering condition can be stated as

$$
C_{1}^{j} e(k+j \mid k-1) \leq \sigma\left(\alpha \sum_{i=0}^{j}\left\|x_{k-i+j}\right\|^{\omega}+C_{2}^{j}\right)
$$

and

$$
\begin{aligned}
& C_{1}^{j} e(k+j \mid k-1)-\sigma\left(\alpha \sum_{i=0}^{j}\left\{\left\|x_{k-i+j}\right\|^{\omega}\right\}+C_{2}^{j}\right) \leq \\
& C_{1}^{j-1} e(k+j-1 \mid k-1)-\sigma\left(\alpha \sum_{i=0}^{j-1}\left\{\left\|x_{k-i+j}\right\|^{\omega}\right\}+C_{2}^{j-1}\right)
\end{aligned}
$$

and

$$
\left\|K^{*}(k+j)\right\| e(k+j) \leq \gamma^{\mu}
$$

The next OCP is triggered whenever condition (27a) or (27b) or (27c) is violated.

The previous analysis guarantees that the closed loop system will have the same convergence properties as in [14]. However, the OCP in this paper is not calculated at each time instant, but only when the triggering condition is violated. The next theorem can be stated:

Theorem 3: Consider the system (3), subject to (2) under an NMPC strategy and assume that the previously presented Assumption 1 holds. The NMPC control law provided by (10a)-(10b) is triggered whenever condition (27a)-(27c) is violated. Between inter-event times the control law (11) is applied to the system. The overall framework drives the closed loop system towards a compact set where it is ultimately bounded. 


\section{EXAmple}

In this section, a simulated example of the proposed eventbased framework is presented. The system under consideration is a linear system under a quadratic MPC scheme. A comparison is made, between the event-based framework proposed in [5], and the proposed approach where the last computed control sequence is applied to the system along with a correction term given by a perturbation solution of the MPC. For illustrative purposes, the numerical values of the parameters of the system that was taken as in the example section in [6]. The disturbance parameter and the length of the prediction horizon is set to $\|w\| \leq 0.8$ rand and $N=6$, respectively.

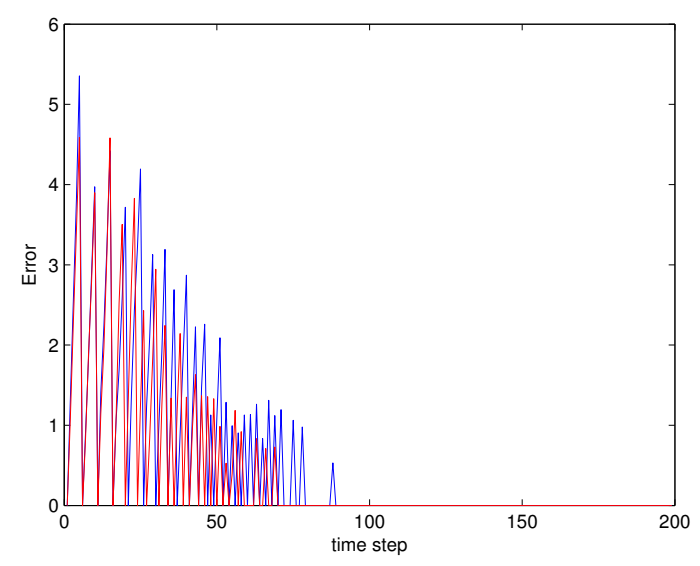

Fig. 1. The error given from (5). The blue line represents the error when the last computed MPC law is applied to the system during the inter-event time-steps, while the red line represents the error when the last computed MPC law is applied to the system in conjunction with a correction term.

The simulation results are reported in Fig. 1 where the error (5) between the real state and the predicted state of the system given by the nominal model (1), is depicted. The error is zero when an event is triggered. From Fig.1 it can be witnessed that both approaches have comparable results and that with both approaches the inter-calculation times are strictly larger than one when the system is away from the equilibrium.

The next Fig.2, depicts a state sequence of the system under the event-based scheme given in [5] and is represented by the blue line. The state sequence of the system when the event-based scheme that exploits the neighboring extremal approach is applied to the system is represented by the red line. As it can be seen in Fig. 2 the system under the proposed event-based approach of this paper, has faster convergence properties.

\section{Conclusions}

In this paper, an event-based framework for the control of a general nonlinear constrained system under NMPC was proposed and analyzed. The event-based formulation consists in triggering the solution of the OCP of the NMPC only when an event occurs. During the inter-event period the control sequence provided from the previous triggering event

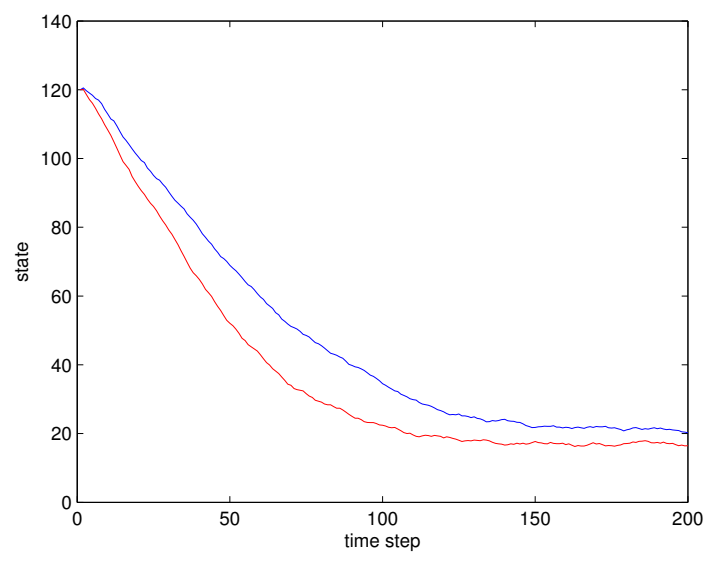

Fig. 2. A state sequence of the system. The blue line represents the state sequence of the system when the last computed MPC law is applied to the system during inter-event time-steps, while the red line represents the state sequence of the system under the proposed approach.

in conjunction with a correction term is used in an open-loop fashion. This event-based scheme is favorable in a number of occasions, because it is possible to reduce the number of times the control law should be computed. This results to the alleviation of the energy consumption. Future work involves formulating the MPC control problem in a self-triggered scheme. With this approach the next control updates are decided at the previous ones, thus, the need for continuous monitoring of the measurement error can be relaxed.

\section{APPENDIX}

The perturbation solution for discrete-time MPC problems with state, control and terminal constraints is derived in this section. The cost function of the optimal control problem of MPC for the nominal system (1), is given by

$$
J_{N}(x(0), u)=\sum_{k=0}^{N-1}\{F(x(k), u(k))\}+V(x(N))
$$

Notice that a simpler notation of the cost function of the OCP, given by (10a) is used. The constraints (10b) are assumed to have the form $C(x(k), u(k)) \leq 0, \psi(x(N))=0$, where $C$ : $\mathbb{R}^{n+m} \rightarrow \mathbb{R}^{l}$ and $\psi: \mathbb{R}^{n} \rightarrow \mathbb{R}^{q}$. This assumption is helpful in the subsequent analysis, but not restrictive to the general constrained case. The subscripts $x, u$ will denote the partial derivatives vector functions.

Next, the standard procedure of [3] and [8] is followed. The augmented performance index obtained by adjoining the constraints, is given by

$$
\bar{J}_{N}=V^{a}(x(N))+\sum_{k=0}^{N-1}\left\{H(x(k))-\lambda(k+1)^{\top} x(k+1)\right\}
$$

where $V^{a}=V(x(N))+v^{\top} \psi(x(N))$ and the Hamiltonian is given by $H(k)=F(x(k), u(k))+\lambda(k+1)^{\top} f(x(k), u(k))+$ $\mu(k)^{\top} C(x(k), u(k))$, where $\lambda(k)$ and $v^{\top}$ are multiplier sequence and a set of $q$ multipliers, respectively. 
The neighboring optimum feedback law with the present deviation from the optimal path $d x(k)$, while minimizing the cost function, is given by

$$
d u(k)=K^{*}(k) d x(k)
$$

with

$$
K^{*}(k) \triangleq Z_{u u}\left(Z_{u x}+Z_{u v} Q^{-1}(k) R^{\top}(k)\right)
$$

We have that

$$
\begin{gathered}
Z_{u u}=\left[f_{u}+K_{2} S(k+1) f_{u}\right]^{-1} \\
Z_{u x}=K_{2} S(k+1) f_{x}-f_{x}+K_{1} \\
Z_{u v}=K_{2} R(k+1)
\end{gathered}
$$

also it can be derived that

$$
\begin{gathered}
K_{1}=f_{x}(k)-\left[\begin{array}{ll}
f_{u}(k) & 0
\end{array}\right]\left[\begin{array}{cc}
H_{u u}(k) & C_{u}^{\top}(k) \\
C_{u}(k) & 0
\end{array}\right]^{-1}\left[\begin{array}{c}
H_{u x}(k) \\
C_{x}(k)
\end{array}\right] \\
K_{2}=-\left[\begin{array}{ll}
f_{u}(k) & 0
\end{array}\right]\left[\begin{array}{cc}
H_{u u}(k) & C_{u}^{\top}(k) \\
C_{u}(k) & 0
\end{array}\right]^{-1}\left[\begin{array}{c}
f_{u}^{\top}(k) \\
0
\end{array}\right] \\
K_{3}=H_{x x}(k)-\left[\begin{array}{ll}
H_{x u}(k) & C_{x}^{\top}(k)
\end{array}\right]\left[\begin{array}{cc}
H_{u u}(k) & C_{u}^{\top}(k) \\
C_{u}(k) & 0
\end{array}\right]^{-1}\left[\begin{array}{c}
H_{u x}(k) \\
C_{x}(k)
\end{array}\right]
\end{gathered}
$$

The terms $S(k), R(k)$ and $Q(k)$ in equations (30) through (32) are given by

$$
\begin{gathered}
S(k)=K_{3}+K_{1}^{\top} S(k+1) f_{x}+K_{1}^{\top} S(k+1) f_{u} Z_{u u} Z_{u x} \\
R(k)=K_{1}^{\top} S(k+1) f_{u} Z_{u u} Z_{u v} \\
Q(k)=R^{\top}(k+1) f_{u} Z_{u u} Z_{u v}+Q(k+1)
\end{gathered}
$$

These recursive equations must satisfy the boundary conditions, namely $S(N)=V_{x x}^{a}, R(N)=\psi_{x}(x(N))$ and $Q(N)=0$.

Lemma 4: The norm of the difference between the real evolution of the system when the control law (11) is applied to the system and the predicted evolution of the system at the same time step satisfies:

$$
\left\|\hat{x}(k+j \mid k-1)-x_{k+j}\right\| \leq L_{f_{x}}^{j} \gamma+L_{m}(j) L_{f_{u}} \gamma^{u}
$$

Lemma 5: It holds that the norm of the difference between $x^{n}(k+j+m \mid k+m)$ and $\hat{x}(k+j+m \mid k-1)$ is bounded. Note, that $x^{n}(\cdot)$, as well as $\hat{x}(\cdot)$ are the "neighboring" state of the system and the predicted one, respectively, and that both are given by the nominal model (1). In particular,

$$
\begin{aligned}
& \left\|x^{n}(k+j+m \mid k+m)-\hat{x}(k+j+m \mid k-1)\right\| \\
& \leq L_{f_{x}}^{j} e(k+m \mid k-1)+L_{m}(j) L_{f_{u}} \gamma^{u}
\end{aligned}
$$

The proof of Lemma 4, Lemma 5 as well as the derivation of (28) are omitted due to space constraints and will be presented in an extended journal version.

\section{REFERENCES}

[1] K. Åström. Event based control. Analysis and Design of Nonlinear Control Systems, pages 127 - 147, 2008.

[2] A. Bemporad, S. Di Cairano, and J. Julvez. Event-based model predictive control and verification of integral continuous-time hybrid automata. HSCC '06:Proc. of the 11th International Conference on Hybrid Systems: Computation and Control, pages 93-107, 2006.

[3] A. E. Bryson and Y.-C. Ho. Applied Optimal Control, Optimization, Estimation, and Control. Hemisphere Publishing Corp., Washington D. C., 1975.

[4] D.V. Dimarogonas and K.H. Johansson. Event-triggered control for multi-agent systems. 48th IEEE Conf. Decision and Control, pages $7131-7136,2009$.

[5] A. Eqtami, D.V. Dimarogonas, and K.J. Kyriakopoulos. Eventtriggered strategies for decentralized model predictive controllers. IFAC World Congress, 2011.

[6] E. Franco, Th. Parisini, and M.M. Polycarpou. Cooperative control of discrete-time agents with delayed information exchange: a receding horizon approach. 43th IEEE Conf. Decision and Control, pages 4274 - 4279, 2004.

[7] E. Garcia and P.J. Antsaklis. Model-based event-triggered control with time-varying network delays. 50th IEEE Conf. Decision and Control \& Eur. Control Conf., pages 1650 - 1655, 2011.

[8] R. Ghaemi, J. Sun, and I. Kolmanovsky. Model predictive control for constrained discrete time systems: An optimal perturbation analysis approach. American Control Conference, pages 3757 - 3762, 2007.

[9] B. Gholami, B.W. Gordon, and C.A. Rabbath. Uncertain nonlinear receding horizon control systems subject to non-zero computation time. 44th IEEE Conf. Decision and Control, pages 3765 - 3770, 2005.

[10] L. Grune and F. Muller. An algorithm for event-based optimal feedback control. 48th IEEE Conf. Decision and Control, pages 5311 $-5316,2009$.

[11] W.P.M.H. Heemels, J.H. Sandee, and P.P.J. Van Den Bosch. Analysis of event-driven controllers for linear systems. International Journal of Control, 81(4):571-590, 2007.

[12] Y. Iino, T. Hatanaka, and M. Fujita. Event-predictive control for energy saving of wireless networked control system. American Control Conference, pages 2236-2242, 2009.

[13] L. Magni, D. Raimondo, and R. Scattolini. Regional input-to-state stability for nonlinear model predictive control. IEEE Trans. Autom. Control, 51:1548-1553, 2006.

[14] D. Limon Marruedo, T. Alamo, and E.F. Camacho. Input-to-state stable mpc for constrained discrete-time nonlinear systems with bounded additive uncertainties. 41st IEEE Conf. Decision and Control, pages $4619-4624,2002$.

[15] A. Molin and S. Hirche. Optimal design of decentralized eventtriggered controllers for large-scale systems with contention-based communication. 50th IEEE Conf. Decision and Control \& Eur. Control Conf., pages $4710-4716,2011$.

[16] G. Pin, D.M. Raimondo, L. Magni, and T. Parisini. Robust model predictive control of nonlinear systems with bounded and statedependent uncertainties. IEEE Transactions on Automatic Control, 54(7): 1681 - 1687, 2009.

[17] J. Sijs, M. Lazar, and W.P.M.H. Heemels. On integration of eventbased estimation and robust mpc in a feedback loop. Proceedings of the 13th ACM international conference on Hybrid systems: computation and control, pages 31-40, 2010.

[18] P. Tabuada. Event-triggered real-time scheduling of stabilizing control tasks. IEEE Transactions on Automatic Control, 52(9):1680-1685, 2007.

[19] P. Varutti, B. Kern, T. Faulwasser, and R. Findeisen. Event-based model predictive control for networked control systems. 48th IEEE Conf. Decision and Control, pages 567 - 572, 2009.

[20] X. Wang and M.D. Lemmon. Event design in event-triggered feedback control systems. 47th IEEE Conf. Decision and Control, pages 21052110, 2008.

[21] L. Wurth, R. Hannemann, and W. Marquardt. Neighboring-extremal updates for nonlinear model-predictive control and dynamic real-time optimization. Journal of Process Control, 19(8):1277-1288, 2009. 